\title{
Effects of Binary Chemical Reaction and Activation Energy on MHD Boundary Layer Heat and Mass Transfer Flow with Viscous Dissipation and Heat Generation/Absorption
}

\author{
Kh. Abdul Maleque \\ American International University-Bangladesh, House 23, 17, Kamal Ataturk Avenue, Banani, Dhaka-1213, Bangladesh \\ Correspondence should be addressed to Kh. Abdul Maleque; maleque@aiub.edu
}

Received 17 December 2012; Accepted 6 January 2013

Academic Editors: G. L. Aranovich, C. D. Daub, P. Espeau, and Z. Xu

Copyright (c) $2013 \mathrm{Kh}$. Abdul Maleque. This is an open access article distributed under the Creative Commons Attribution License, which permits unrestricted use, distribution, and reproduction in any medium, provided the original work is properly cited.

\begin{abstract}
We study an unsteady MHD free convection heat and mass transfer boundary layer incompressible fluid flow past a vertical porous plate in the presence of viscous dissipation, heat generation/absorption, chemical reaction, and Arrhenius activation energy. The plate is moving with uniform velocity. The chemical reaction rate in the function of temperature is also considered. The governing partial differential equations are reduced to ordinary differential equations by introducing local similarity transformation (Maleque (2010)) and then are solved numerically by shooting method using the Nachtsheim-Swigert iteration technique. The results of the numerical solution are then presented graphically as well as the tabular form for difference values of the various parameters.
\end{abstract}

\section{Introduction}

In free convection boundary layer flows with simultaneous heat mass transfer, one important criterion that is generally not encountered is the species chemical reactions with finite Arrhenius activation energy. The Arrhenius law is usually of the following form [1]:

$$
K=B\left(T-T_{\infty}\right)^{w} \exp \left[\frac{-E_{a}}{k\left(T-T_{\infty}\right)}\right],
$$

where $K$ is the rate constant of chemical reaction and $B$ is the preexponential factor, simply prefactor (constant), is based on the fact that increasing the temperature frequently causes a marked increase in the rate of reactions. $E_{a}$ is the activation energy and $k=8.61 \times 10^{-5} \mathrm{eV} / \mathrm{K}$ is the Boltzmann constant which is the physical constant relating energy at the individual particle level with temperature observed at the collective or bulk level.

In areas such as geothermal or oil reservoir engineering, the above phenomenon is usually applicable. Apart from experimental works in these areas, it is also important to make some theoretical efforts to predict the effects of the activation energy in flows mentioned above. But in this regard the very few theoretical works are available in the literature.
The reason is that the chemical reaction processes involved in the system are quite complex and generally the mass transfer equation that is required for all the reactions involved also becomes complex. Theoretically, such an equation is rather impossible to tackle. From chemical kinetic viewpoint, this is a very difficult problem, but if the reaction is restricted to binary type a lot of progress can be made. The thermomechanical balance equations for a mixture of general materials were first formulated by Truesdell $[2,3]$. Thereafter, Mills [4] and Beevers and Craine [5] have obtained some exact solutions for the boundary layer flow of a binary mixture of incompressible Newtonian fluids. Several problems relating to the mechanics of oil and water emulsions, particularly with regard to applications in lubrication practice, have been considered within the context of a binary mixture theory by Al-Sharif et al. [6] and Wang et al. [7].

A simple model involving binary reaction was studied by Bestman [8]. He considered the motion through the plate to be large which enabled him to obtain analytical solutions (subject to same restrictions) for various values of activation energy by employing the perturbation technique proposed by Singh and Dikshit [9]. Bestman [10] and Alabraba et al. [11] took into account the effect of the Arrhenius activation energy under the different physical conditions. 
Recently Kandasamy et al. [12] studied the combined effects of chemical reaction, heat and mass transfer along a wedge with heat source, and concentration in the presence of suction or injection. Their result shows that the flow field is influenced appreciably by chemical reaction, heat source, and suction or injection at the wall of the wedge. Recently, Makinde et al. [13] studied the problem of unsteady convection with chemical reaction and radiative heat transfer past a flat porous plate moving through a binary mixture in an optically thin environment. More recently Maleque [14] studied the effects of exothermic chemical reaction and activation energy on free convective heat and mass transfer flow past a vertical plate. In the present paper, we investigate a numerical solution of unsteady natural convection heat and mass transfer boundary layer flow past a vertical porous plate taking into account the effects of viscous dissipation, heat generation/absorption, and chemical reaction with Arrhenius activation energy in the presence of uniform magnetic field. The plate is moving with uniform velocity. The chemical reaction rate in the function of temperature is also considered. The governing partial differential equations are reduced to ordinary differential equations by introducing local similarity transformation (Maleque [15]). Numerical solutions to the reduced nonlinear similarity equations are then obtained by adopting Runge-Kutta and shooting methods using the Nachtsheim-Swigert iteration technique. The results of the numerical solution are then presented graphically as well as the tabular form for difference values of the various parameters.

\section{Governing Equations}

We consider the boundary wall to be of infinite extension so that all quantities are homogeneous in $x$ and hence all derivatives with respect to $x$ are neglected. The $x$-axis is taken along the plate and $y$-axis is perpendicular to the plate. Assuming a uniform magnetic field, $B_{0}$ is applied perpendicular to the plate and the plate is moving with uniform velocity $U_{0}$ in its own plane. The system of governing equations in the presence of viscous dissipation, heat generation/absorption, and a binary chemical reaction with Arrhenius activation energy is of the form

$$
\begin{gathered}
\frac{\partial v}{\partial y}=0 \\
\frac{\partial u}{\partial t}+v \frac{\partial u}{\partial y}=v \frac{\partial^{2} u}{\partial y^{2}}+g \beta\left(T-T_{\infty}\right) \\
+g \beta^{*}\left(C-C_{\infty}\right)-\frac{\sigma B_{0}^{2}}{\rho} u \\
\rho c_{p}\left(\frac{\partial T}{\partial t}+v \frac{\partial T}{\partial y}\right)=\kappa \frac{\partial^{2} T}{\partial y^{2}}+\mu\left(\frac{\partial u}{\partial y}\right)^{2}+Q_{0}\left(T-T_{\infty}\right) \\
\frac{\partial C}{\partial t}+v \frac{\partial C}{\partial y} \\
=D_{m} \frac{\partial^{2} C}{\partial y^{2}}-k_{r}^{2}\left(T-T_{\infty}\right)^{w} \exp \left[\frac{-E_{a}}{k\left(T-T_{\infty}\right)}\right]\left(C-C_{\infty}\right)
\end{gathered}
$$

The boundary conditions of the above system are

$$
\begin{aligned}
& u=U_{0}, \quad v=v(t), \quad T=T_{w}, \quad C=C_{w} \quad \text { at } y=0, \\
& u=0, \quad T \longrightarrow T_{\infty}, \quad C \longrightarrow C_{\infty}, \quad \text { at } y=\infty,
\end{aligned}
$$

where $(u, v)$ is the velocity vector, $T$ is the temperature, $C$ is the concentration of the fluid, $v$ is the kinematic coefficient of viscosity, $\kappa$ is the heat diffusivity coefficient, $\beta$ and $\beta^{*}$ are the coefficients of volume expansions for temperature and concentration, respectively, $D_{m}$ is the coefficient of mass diffusivity, $k_{r}^{2}$ is the chemical reaction rate constant, and ( $T$ $\left.T_{\infty}\right)^{w} \exp \left[-E_{a} / k\left(T-T_{\infty}\right)\right]$ is the Arrhenius function where $w$ is a unit less constant exponent fitted rate constants typically lie in the range $-1<w<1$.

\section{Mathematical Formulations}

In order to solve the governing equations (2) to (5) under the boundary conditions (6), we adopt the well-defined similarity technique to obtain the similarity solutions. For this purpose the following nondimensional variables are now introduced:

$$
\begin{array}{cc}
\eta=\frac{y}{\delta(t)}, & \frac{u}{U_{0}}=f(\eta), \\
\frac{T-T_{\infty}}{T_{w}-T_{\infty}}=\theta(\eta), & \frac{C-C_{\infty}}{C_{w}-C_{\infty}}=\phi(\eta) .
\end{array}
$$

From the equation of continuity (2), we have

$$
v(t)=-\frac{v_{0} v}{\delta(t)},
$$

where $v_{0}$ is the dimensionless suction/injection velocity at the plate, $v_{0}>0$ corresponds to suction, and $v_{0}<0$ corresponds to injection.

Introducing the dimensionless quantities from (7) and $v$ from (8) in (3), (4), and (5), we finally obtain the nonlinear ordinary differential equations as

$$
\begin{gathered}
f^{\prime \prime}+\left(\eta \frac{\delta \delta^{\prime}}{v}+v_{0}\right) f^{\prime}-M f+G_{r} \theta+G_{m} \phi=0, \\
P_{r}^{-1} \theta^{\prime \prime}+\left(\eta \frac{\delta \delta^{\prime}}{v}+v_{0}\right) \theta^{\prime}+\gamma \theta+E_{c} f^{\prime 2}=0 \\
S_{c}^{-1} \phi^{\prime \prime}+\left(\eta \frac{\delta \delta^{\prime}}{v}+v_{0}\right) \phi^{\prime}-\lambda^{2} e^{-E / \theta} \theta^{w} \phi=0 .
\end{gathered}
$$

Here, Grashof number $G_{r}=g \beta\left(T_{w}-T_{\infty}\right) \delta^{2} / v U_{0}$, modified (Solutal) Grashof number $G_{m}=g \beta^{*}\left(C_{w}-C_{\infty}\right) \delta^{2} / v U_{0}$, Prandtl number $P_{r}=\rho v c_{p} / \kappa$, magnetic parameter $M=$ $\sigma^{\prime} \delta^{2} B_{0}^{2} / \mu$, Eckert number $E_{c}=U_{0}^{2} / \Delta T c_{p}$, the chemical reaction rate constant $\lambda^{2}=k_{r}^{2} \delta^{2}\left(T_{w}-T_{\infty}\right)^{w} / v$, Schmidt number $S_{c}=v / D_{m}$, the nondimensional activation energy $E=$ $E_{a} / k\left(T_{w}-T_{\infty}\right)$, the dimensionless heat generation, or absorption coefficient $\gamma=\delta^{2} Q_{0} / v \rho c_{p}$.

Equations (9) to (11) are similar except for the term $\delta \delta^{\prime} / v$, where time $t$ appears explicitly. Thus, the similarity 
condition requires that $\delta \delta^{\prime} / v$ must be a constant quantity. Hence, following Maleque [15], one can try a class of solutions of (9) to (11) by assuming the following:

$$
\frac{\delta \delta^{\prime}}{v}=A(\text { Constant }) \text {. }
$$

From (12) we have

$$
\delta(t)=\sqrt{2 A v t+L^{2}}
$$

where the constant of integration $L$ is determined through the condition that $\delta=L$ when $t=0$. Here $A=0$ implies that $\delta=$ $L$ represents the length scale for steady flow and $A \neq 0$, that is, $\delta$ represents the length scale for unsteady flow. Since $\delta$ is a scaling factor as well as similarity parameter, any other values of $A$ in (13) would not change the nature of the solution except that the scale would be different. Finally introducing (12) in (9) to (11), respectively, we have the following dimensionless nonlinear ordinary differential equations:

$$
\begin{gathered}
f^{\prime \prime}+\left(\eta A+v_{0}\right) f^{\prime}-M f+G_{r} \theta+G_{m} \phi=0, \\
P_{r}^{-1} \theta^{\prime \prime}+\left(\eta A+v_{0}\right) \theta^{\prime}+\gamma \theta+E_{c} f^{\prime 2}=0, \\
S_{c}^{-1} \phi^{\prime \prime}+\left(A \eta+v_{0}\right) \phi^{\prime}-\lambda^{2} \exp \left(-\frac{E}{\theta}\right) \theta^{w} \phi=0 .
\end{gathered}
$$

The boundary condition equation (6) then becomes

$$
\begin{aligned}
f(0) & =1, & \theta(0) & =1, & & \phi(0)=1, \\
f(\infty) & =0, & \theta(\infty) & =0, & \phi(\infty) & =0 .
\end{aligned}
$$

In all equations primes denote the differentiation with respect to $\eta$. Equations (14) to (16) are solved numerically under the boundary conditions (17) using Nachtsheim-Swigert iteration technique.

\section{Numerical Solutions}

Equations (14)-(16) are solved numerically under the boundary conditions (17) using Nachtsheim Swigert [16] iteration technique. In (17), there are three asymptotic boundary conditions and hence follows three unknown surface conditions $f^{\prime}(0), \theta^{\prime}(0)$, and $\phi^{\prime}(0)$. Within the context of the initial-value method and the Nachtsheim-Swigert iteration technique, the outer boundary conditions may be functionally represented by the first-order Taylor's series as follows:

$$
\begin{aligned}
f\left(\eta_{\max }\right) & =f(X, Y, Z) \\
& =f_{c}\left(\eta_{\max }\right)+\Delta X f_{X}+\Delta Y f_{Y}+\Delta Z f_{Z}=\delta_{1}, \\
\theta\left(\eta_{\max }\right) & =\theta(X, Y, Z) \\
& =\theta_{c}\left(\eta_{\max }\right)+\Delta X \theta_{X}+\Delta Y \theta_{Y}+\Delta Z \theta_{Z}=\delta_{2}, \\
\phi\left(\eta_{\max }\right) & =\phi(X, Y, Z) \\
& =\phi_{c}\left(\eta_{\max }\right)+\Delta X \phi_{X}+\Delta Y \phi_{Y}+\Delta Z \phi_{Z}=\delta_{3},
\end{aligned}
$$

with the asymptotic convergence criteria given by

$$
\begin{aligned}
f^{\prime}\left(\eta_{\max }\right) & =f^{\prime}(X, Y, Z) \\
& =f_{c}^{\prime}\left(\eta_{\max }\right)+\Delta X f_{X}^{\prime}+\Delta Y f_{Y}^{\prime}+\Delta Z f_{Z}^{\prime}=\delta_{4}, \\
\theta^{\prime}\left(\eta_{\max }\right) & =\theta^{\prime}(X, Y, Z) \\
& =\theta_{c}^{\prime}\left(\eta_{\max }\right)+\Delta X \theta_{X}^{\prime}+\Delta Y \theta_{Y}^{\prime}+\Delta Z \theta_{Z}^{\prime}=\delta_{5}, \\
\phi^{\prime}\left(\eta_{\max }\right) & =\phi^{\prime}(X, Y, Z) \\
& =\phi_{c}^{\prime}\left(\eta_{\max }\right)+\Delta X \phi_{X}^{\prime}+\Delta Y \phi_{Y}^{\prime}+\Delta Z \phi_{Z}^{\prime}=\delta_{6},
\end{aligned}
$$

where $X=f^{\prime}(0), Y=\theta^{\prime}(0), Z=\phi^{\prime}(0)$, and $X, Y, Z$ subscripts indicate partial differentiation, for example, $f_{Y}^{\prime}=$ $\partial f^{\prime}\left(\eta_{\max }\right) / \partial \theta^{\prime}(0)$. The subscript $c$ indicates the value of the function at $\eta_{\max }$ to be determined from the trial integration.

Solutions of these equations in a least-square sense requires determining the minimum value of $E=\delta_{1}^{2}+$ $\delta_{2}^{2}+\delta_{3}^{2}+\delta_{4}^{2}+\delta_{5}^{2}+\delta_{6}^{2}$ with respect to $X, Y$, and $Z$. To solve $\Delta X, \Delta Y$ and $\Delta Z$ we require to differentiate $E$ with respect to $X, Y$, and $Z$ respectively. Thus, adopting this numerical technique, a computer program was set up for the solutions of the basic nonlinear differential equations of our problem, where the integration technique was adopted as the six ordered Range-Kutta method of integration. The results of this integration are then displayed graphically in the form of velocity, temperature, and concentration profiles in Figures 1-11. In the process of integration, the local skinfriction coefficient, the local rates of heat, and mass transfer to the surface, which are of chief physical interest, are also calculated out. The equation defining the wall skin-friction is

$$
\tau=-\mu\left(\frac{\partial u}{\partial y}\right)_{y=0} .
$$

Hence, the skin-friction coefficient is given by

$$
C_{f}=\frac{\tau}{\rho U_{0}^{2}} \alpha-f^{\prime}(0)
$$

The heat flux $\left(q_{w}\right)$ and the mass flux $\left(M_{w}\right)$ at the wall are given by

$$
\begin{gathered}
q_{w}=-\kappa\left(\frac{\partial T}{\partial y}\right)_{y=0}, \\
M_{w}=-D_{M}\left(\frac{\partial C}{\partial y}\right)_{y=0} .
\end{gathered}
$$

Hence the Nusselt number $\left(N_{u}\right)$ and the Sherwood number $\left(S_{h}\right)$ are obtained as

$$
N_{u} \alpha-\theta^{\prime}(0), \quad S_{h} \alpha-\phi^{\prime}(0) .
$$

These above coefficients are then obtained from the procedure of the numerical computations and are sorted in Table 1. 
TABLE 1: Effects of $G_{r}, \lambda, w, \gamma, E$, and $v_{0}$ on the skin friction coefficient, the Nusselt number, and the Sherwood number for $G_{r}=$ $10, G_{m}=1, P_{r}=0.71, v_{0}=3, S_{c}=0.6, \lambda=5, E=1, w=$ $1, \quad M=E_{c}=0$, and $\gamma=0$.

\begin{tabular}{|c|c|c|c|}
\hline & $-f^{\prime}(0)$ & $-\theta^{\prime}(0)$ & $-\phi^{\prime}(0)$ \\
\hline \multicolumn{4}{|l|}{$G_{r}=$} \\
\hline-10.00000 & 6.28618 & 2.58611 & 2.83618 \\
\hline-5.00000 & 4.71973 & 2.59209 & 2.83672 \\
\hline 0.00000 & 3.19774 & 2.59299 & 2.83998 \\
\hline 5.00000 & 1.70224 & 2.59852 & 2.83923 \\
\hline 10.00000 & 0.24525 & 2.60349 & 2.83849 \\
\hline \multicolumn{4}{|l|}{$\lambda=$} \\
\hline 1.00000 & 0.24033 & 2.60608 & 2.26552 \\
\hline 5.00000 & 0.24525 & 2.60349 & 2.83849 \\
\hline 10.00000 & 0.30840 & 2.60610 & 4.37298 \\
\hline \multicolumn{4}{|l|}{$w=$} \\
\hline-0.750000 & 0.06827 & 2.58234 & 3.21792 \\
\hline 0.00000 & 0.16991 & 2.59250 & 3.01768 \\
\hline 0.75000 & 0.20504 & 2.59811 & 2.87688 \\
\hline \multicolumn{4}{|l|}{$E=$} \\
\hline 0.00000 & 0.34340 & 2.60624 & 4.35339 \\
\hline 0.50000 & 0.16287 & 2.58891 & 3.37658 \\
\hline 1.00000 & 0.24525 & 2.60349 & 2.83849 \\
\hline 5.00000 & 0.10981 & 2.58889 & 2.26497 \\
\hline \multicolumn{4}{|l|}{$v_{0}=$} \\
\hline-3.00000 & -3.88025 & 0.10081 & 1.62314 \\
\hline 0.00000 & -3.66969 & 0.95110 & 1.95706 \\
\hline 3.00000 & 0.24525 & 2.60349 & 2.83849 \\
\hline \multicolumn{4}{|l|}{$\gamma=$} \\
\hline-4.00000 & 0.68965 & 3.31240 & 2.75431 \\
\hline-2.00000 & 0.41067 & 2.98095 & 2.78286 \\
\hline 0.00000 & 0.24525 & 2.60349 & 2.83849 \\
\hline 2.00000 & -0.48425 & 2.05361 & 2.92446 \\
\hline 4.00000 & -0.77199 & 1.46498 & 3.02587 \\
\hline
\end{tabular}

\section{Results and Discussions}

The parameters entering into the fluid flow are Grashof number $G_{r}$, Solutal (modified) Grashof number $G_{m}$, magnetic interaction parameter $M$, suction parameter $v_{0}$, Prandtl number $P_{r}$, Eckert number $E_{c}$, the dimensionless heat generation/absorption coefficient $\gamma$, the nondimensional chemical reaction rate constant $\lambda^{2}$, Schmidt number $S_{c}$ and the nondimensional activation energy $E$.

It is, therefore, pertinent to inquire the effects of variation of each of them when the others are kept constant. The numerical results are thus presented in the form of velocity profiles, temperature profiles, and concentration profiles in Figures 1-11 for the different values of $G_{r}, G_{m}, M, E_{c}, \gamma, \lambda, E$, and $v_{0}$. The values of $G_{r}$ are taken to be large $\left(G_{r}=10\right)$ since the value corresponds to a cooling problem that is generally encountered in nuclear engineering in connection with the cooling of reactors. In air $\left(P_{r}=0.71\right)$ the diluting chemical species of most common interests have Schmidt number in

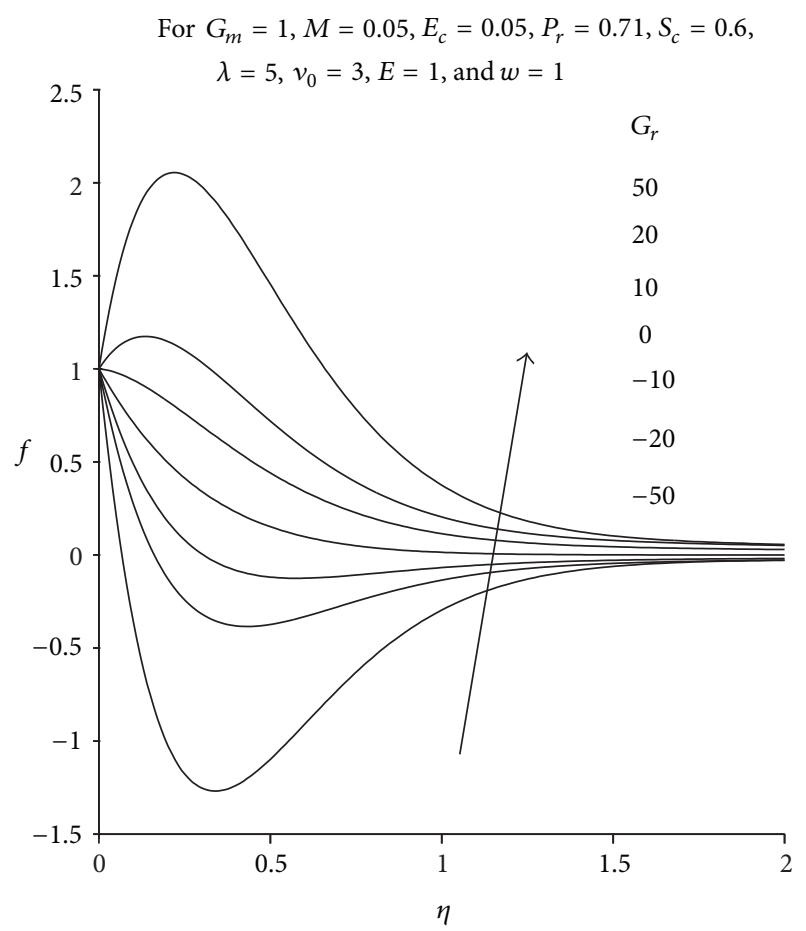

FIGURE 1: Effects of $G_{r}$ on the velocity profiles.

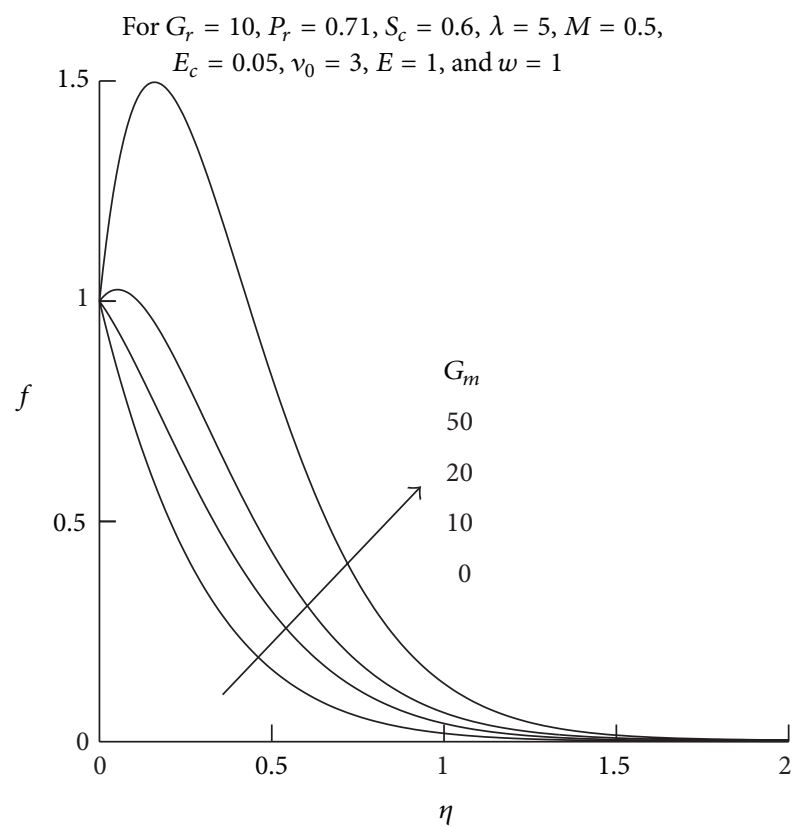

FIGURE 2: Effects of $G_{m}$ on the velocity profiles.

the range from 0.6 to 0.75 . Therefore, the Schmidt number $S_{c}=0.6$ is considered. In particular, 0.6 corresponds to water vapor that represents a diffusing chemical species of the most common interest in air. The values of the suction parameter $v_{0}$ are taken to be large. Apart from the above figures and tables, the representative velocity, temperature and concentration 


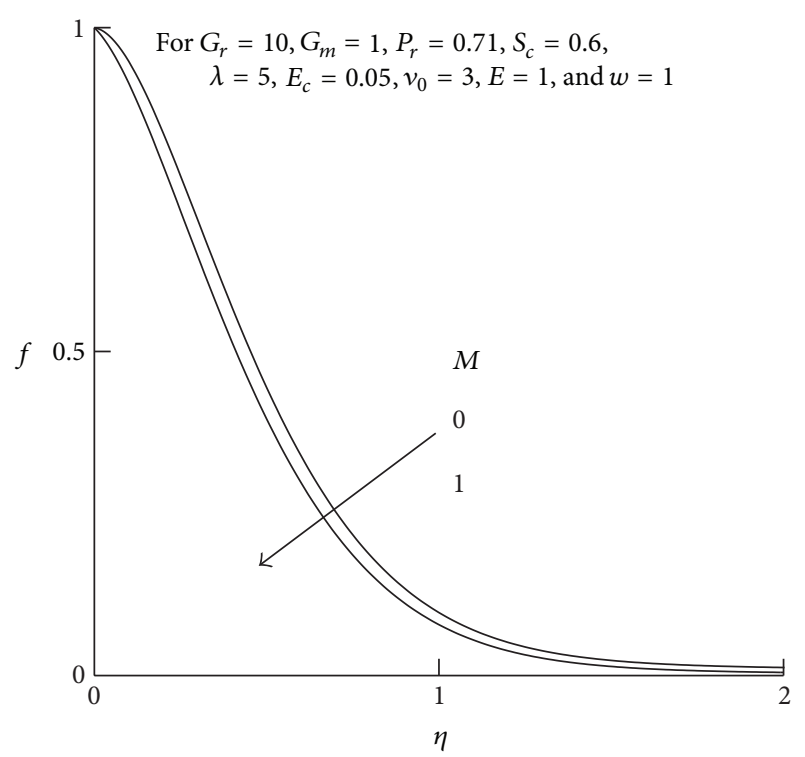

FIGURE 3: Effects of $M$ on the velocity profiles.

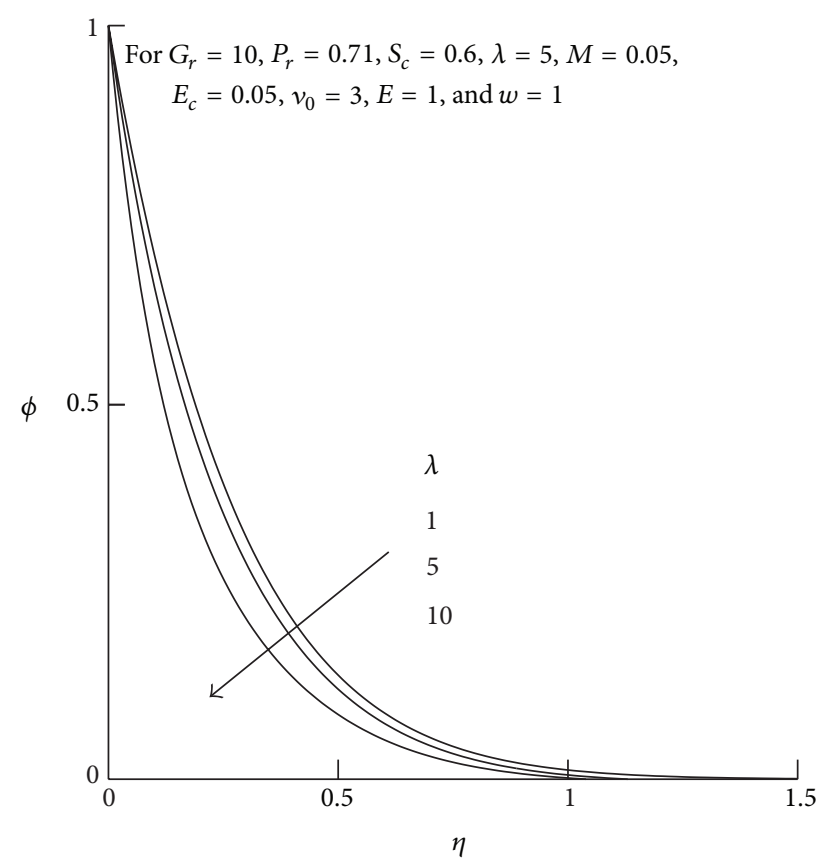

FIgURE 4: Effects of $\lambda$ on the concentration profiles.

profiles, and the values of the physically important parameters, that is, the local shear stress, the local rates of heat, and mass transfers, are illustrated for uniform wall temperature and species concentration in Figures 1-11 and in Table 1.

5.1. Effects of $G_{r}, G_{m}$, and $M$ on the Velocity Profiles. The value of $G_{r}$ is taken to be both positive and negative, since these values represent, respectively, cooling and heating of the plate.

The velocity profiles generated due to impulsive motion of the plate are plotted in Figure 1 for both cooling $\left(G_{r}>0\right)$ and heating $\left(G_{r}<0\right)$ of the plate keeping other parameters

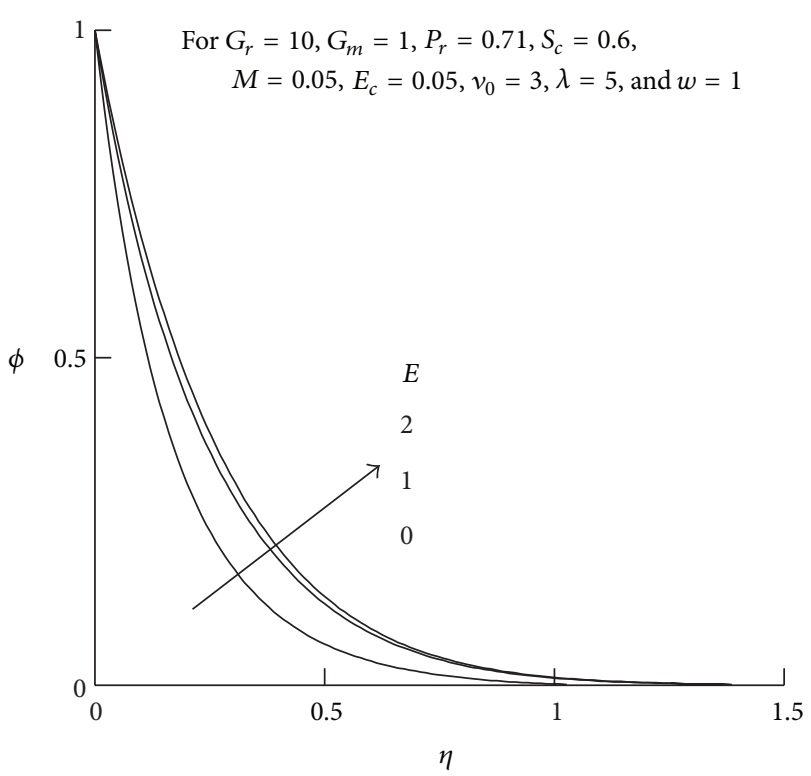

FIGURE 5: Effects of $E$ on the concentration profiles.

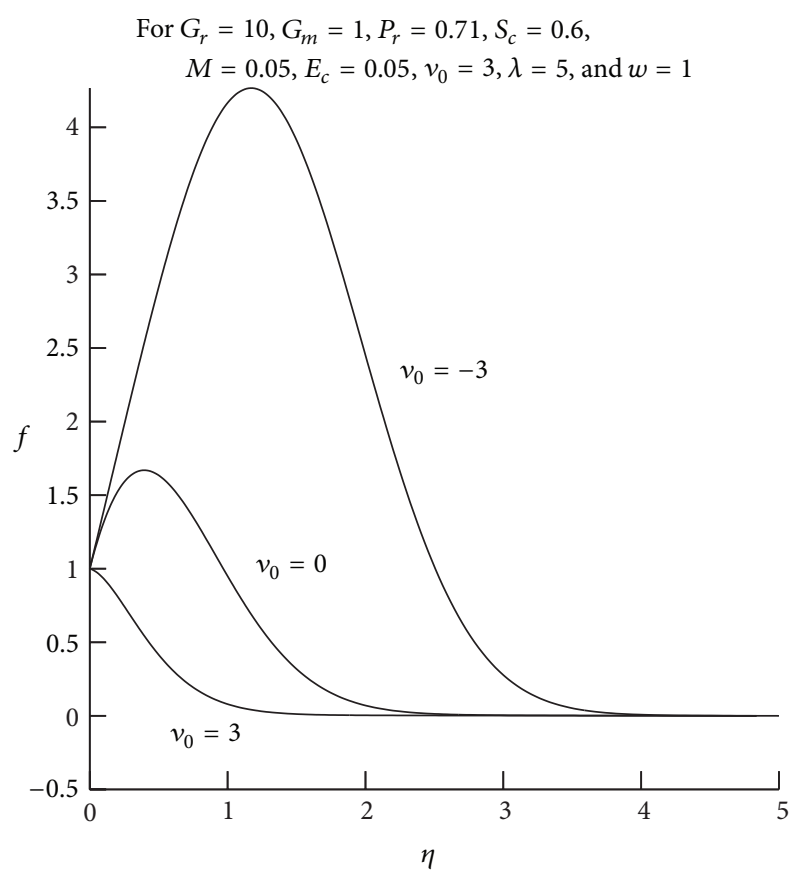

FIGURE 6: Effects of $v_{0}$ on the velocity profiles.

fixed $\left(G_{m}=1.0, M=E_{c}=0.5, P_{r}=0.71, S_{c}=0.6, \lambda=5\right.$, $v_{0}=3.0, E=1.0$, and $\left.w=1\right)$. In Figure 1 , velocity profiles are shown for different values of $G_{r}$. We observe that velocity increases with increasing values of $G_{r}$ for the cooling of the plate. From this figure, it is also observed that the negative increase in the Grashof number leads to the decrease in the velocity field. That is, for heating of the plate (Figure 1), the effects of the Grashof number $G_{r}$ on the velocity field have also opposite effects, as compared to the cooling of the plate.

Solutal Grashof number $G_{m}>0$ corresponds to the fact that the chemical species concentration in the free stream region is less than the concentration at the boundary surface. 


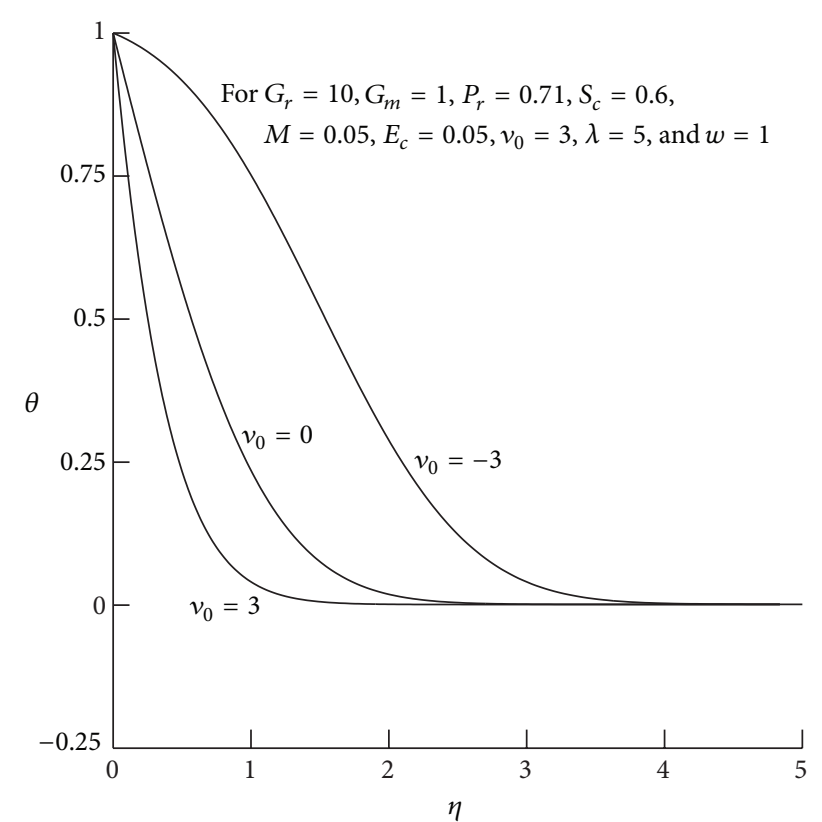

FIGURE 7: Effects of $v_{0}$ on the temperature profiles.

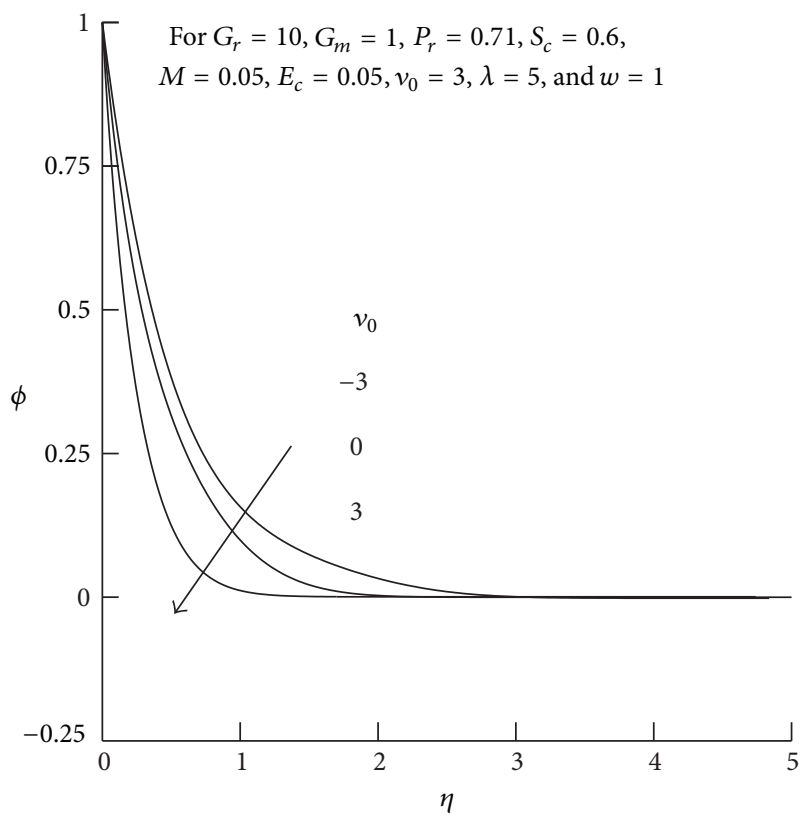

FIGURE 8: Effects of $v_{0}$ on the concentration profiles.

Figure 2 presents the effects of Solutal Grashof number $G_{m}$ on the velocity profiles. It is observed that the velocity profile increases with the increasing values of Solutal Grashof number $G_{m}$.

Imposition of a magnetic field to an electrically conducting fluid creates a drag-like force called Lorentz force. The force has the tendency to slow down the flow around the plate at the expense of increasing its temperature. This is depicted by decreases in velocity profiles as magnetic parameter $M$ increases as shown in Figure 3.

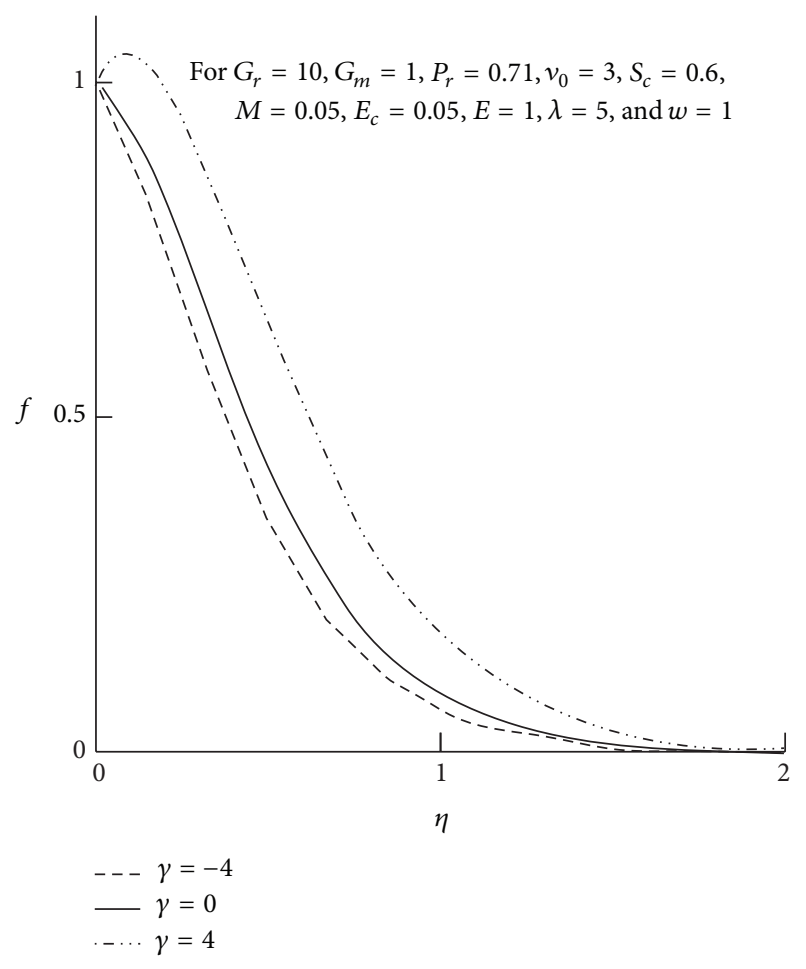

FIGURE 9: Effects of $\gamma$ on the velocity profiles.

5.2. Effects of $\lambda$ and Activation Energy $(E)$ on the Concentration Profiles. Considering chemical reaction rate constant $\lambda^{2}$ is always positive. Figure 4 represents the effect of chemical rate constant $\lambda$ on the concentration. We observe from the last part of (18) that $\lambda^{2} \exp (-E / \theta)$ increases with the increasing values of $\lambda$. We also observe from this equation that increase in $\lambda^{2} \exp (-E / \theta)$ means increase in $\lambda$, leads to the decrease in the concentration profiles. This is in great agreement with Figure 3. The parameter $\lambda$ does not enter directly into the momentum equation but its influence comes through the mass equation. $\lambda$ has no mark effect on the velocity and temperature profiles.

In chemistry, activation energy is a term introduced in 1889 by the Swedish scientist Svante Arrhenius which is defined as the energy that must be overcome for a chemical reaction to occur. Activation energy may also be defined as the minimum energy required starting a chemical reaction. The activation energy of a reaction is usually denoted by $E_{a}$ and given in units of kilojoules per mole.

Activation energy can be thought of as the height of the potential barrier (sometimes called the energy barrier) separating two minima of potential energy (of the reactants and products of a reaction). For a chemical reaction to proceed at a reasonable rate, there should exist an appreciable number of molecules with energy equal to or greater than the activation energy. Effects of activation energy $(E)$ on the concentration are shown in Figure 5. From (1), we observe that chemical reaction rate $(K)$ decreases with the increasing values of activation energy $\left(E_{a}\right)$. We also observe from (18) that increase in activation energy $(E)$ leads to decrease in 


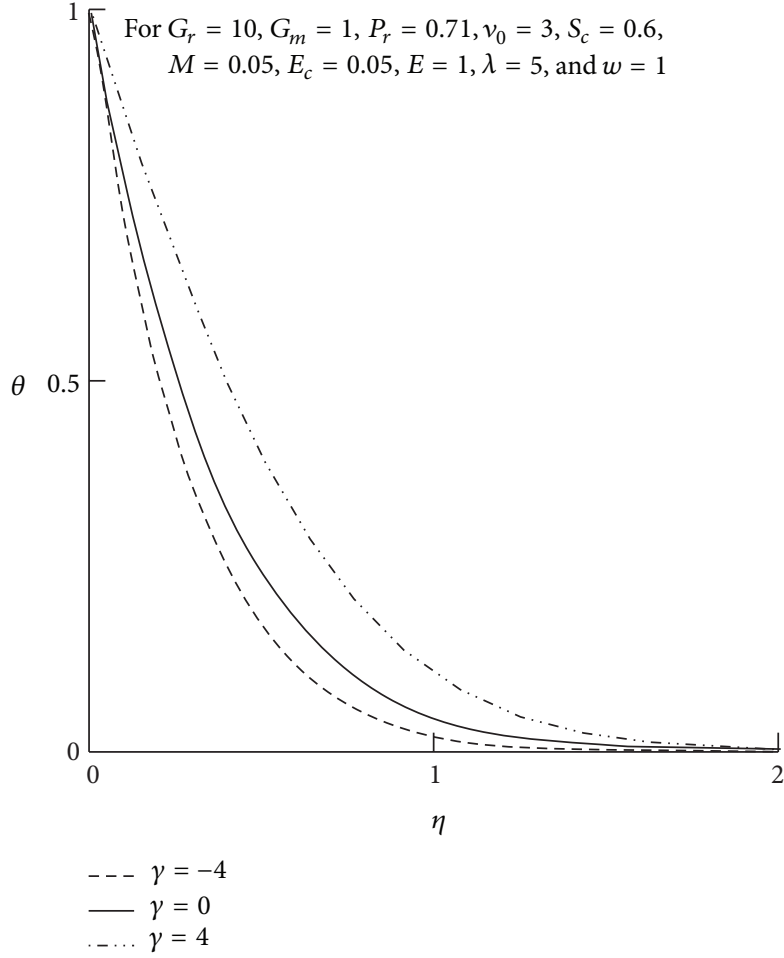

FIGURE 10: Effects of $\gamma$ on the temperature profiles.

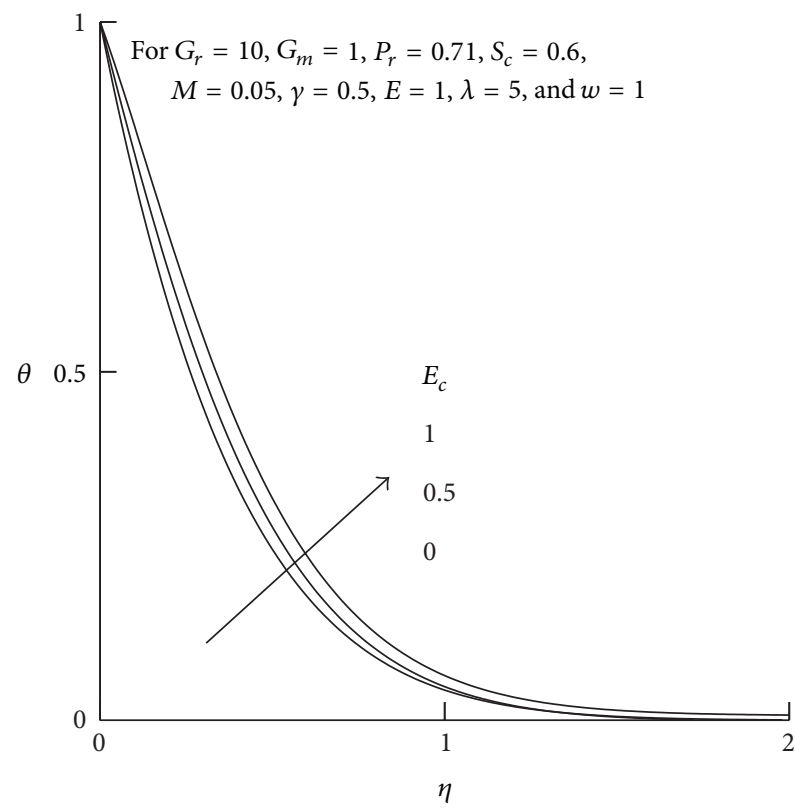

FIGURE 11: Effects of $E_{c}$ on the temperature profiles.

$\lambda^{2} \exp (-E / \theta)$ as well as increase in the concentration profiles shown in Figure 5.

5.3. Effects of Suction/Injection $\left(v_{0}\right)$ on the Velocity, the Temperature, and the Concentration Profiles. The effects of suction and injection $\left(v_{0}\right)$ for $\lambda=5, E=1, G_{r}=5, G_{m}=1, S_{c}=0.6$, and $P_{r}=0.7$ on the velocity profiles, temperature profiles and

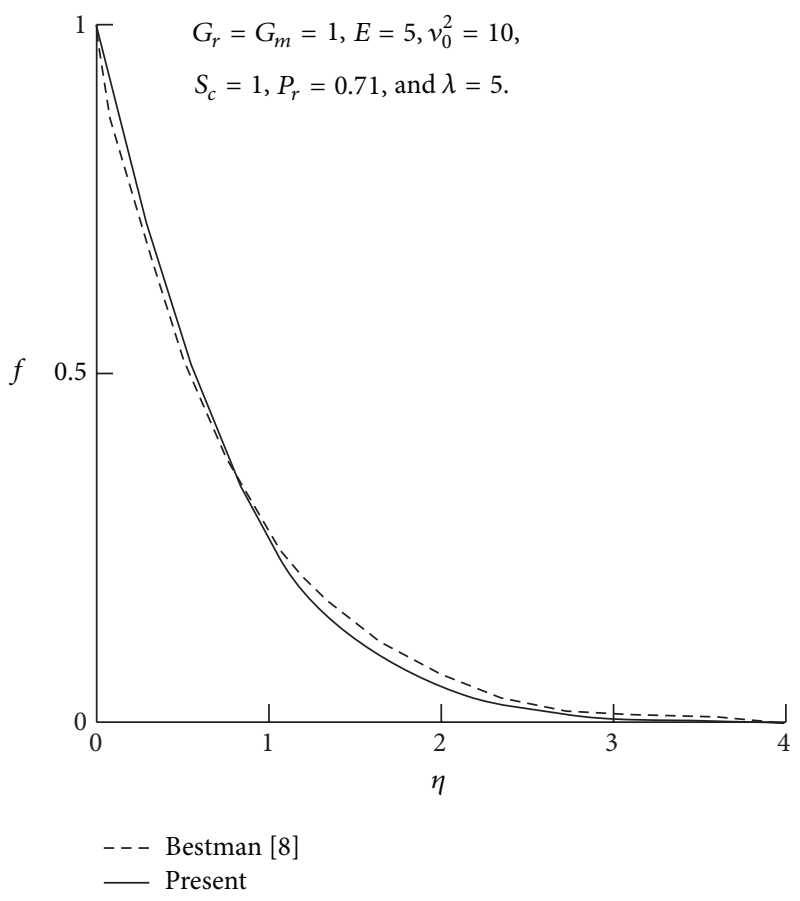

FIGURE 12: Comparison of our calculated velocity profile and the velocity profile of Bestman [8].

concentration profiles are shown, respectively, in Figures 6 to 8 . For strong suction $\left(v_{0}>0\right)$, the velocity, the temperature, and the concentration profiles decay rapidly away from the surface. The fact that suction stabilizes the boundary layer is also apparent from these figures. As for the injection $\left(v_{0}<0\right)$, from Figures 6 to 8 it is observed that the profiles overshoot and are close to the boundary. From Figure 6 it is observed that for strong suction, the velocity is small while for injection the boundary layer is increasingly blown away from the surface of the plate to form an interlayer between the injection and the outer flow regions. Figure 6 also depicts that for high values of injection parameter $\left(v_{0}<0\right)$, the velocity near the plate (for small values of $\eta$ ) is lower than that for smaller values of injection parameter $\left(v_{0}<0\right)$. Figure 7 shows the usual decay of temperature for strong suction while the thermal boundary layer increases rapidly for strong injection. It is seen from Figure 8 that the species concentration within the boundary layer is higher for injection and lower for suction.

5.4. Effects of the Heat Generation and Absorption $(\gamma)$ on the Velocity and the Temperature Profiles. The positive value and the negative value of $\gamma$ represent the heat generation and heat absorption, respectively. The dimensionless heat generation/absorption coefficient $\gamma$ does not enter directly into the equation of continuity, Navier Stokes equations and concentration equation its influence come through the energy equation. In the presence of the buoyancy force, it appears that the heat generation/absorption coefficient $\gamma$ has a marked effect on the boundary layer and the velocity profiles of the fluid is shown in Figure 9. From this figure it has been observed that the effect of heat generation coefficient 
is to expand the boundary layer thickness and opposite effect is found for the effect of heat absorption. Thus, the dimensionless heat generation/absorption coefficient has the same effects on skin-friction coefficients shown in Table 1.

The effects of heat absorption coefficient $(\gamma<0)$ on temperature profiles and the Nusselt number are shown in Figure 10 and Table 1. In general, the effect of heat absorption coefficient $(\gamma<0)$ is to reduce the thickness of the thermal boundary layer. The temperature profiles decrease as the heat absorption coefficient $\gamma(<0)$ increases as shown in Figure 10. On the other hand, the heat generation coefficient $\gamma(<0)$ leads to increasing the temperature profiles. Thermal boundary layer thickness expands for increasing values of the heat generation coefficient $\gamma(>0)$ as shown in Figure 10.

Finally, the effects of heat generation/absorption coefficient $(\gamma)$ on the dimensionless Nusselt number $N_{u}$ are shown in Table 1. The Nusselt number increases with the increasing values of the heat absorption coefficient $(\gamma<$ $0)$. Because the heat absorption increases, the disk tends to supply more energy through its wall to maintain the constant temperature of the wall, and hence the Nusselt number increases. Opposite effects are found for the increasing values of the heat generation coefficient $(\gamma>0)$.

5.5. Effects of the Eckert Number $E_{c}$ on the Temperature Profiles. Figure 11 presents the effects of Eckert number $\left(E_{c}\right)$ on the temperature profiles for $G_{r}=10, G_{m}=1.0, E=$ $1, w=1, \lambda=5, M=0.05, \gamma=0.5, v_{o}=3$, and $P_{r}=0.71$. From this figure it has been observed that the parameter $E_{c}$ has marked effect on the temperature profiles. The temperature profiles increase for increasing values of $E_{c}$. The dimensionless Eckert number $\left(E_{c}\right)$ does not enter directly into the equation of continuity and momentum equation and concentration equation, its influence come through the energy equation. The Eckert number $\left(E_{c}\right)$ has no marked effect on velocity profiles and concentration profiles.

5.6. The Skin-Friction, the Heat Transfer, and the Mass Transfer Coefficients. The skin-friction, the heat transfer, and the mass transfer coefficients are tabulated in Table 1 for different values of $G_{r}, \lambda, E, w, v_{0}$, and $\gamma$. We observe from Table 1 that the skin-friction coefficient $\left(-f^{\prime}(0)\right)$ decreases with increase in the Grash of number $G_{r}$. It is also observed from Table 1 that the rate of mass transfer coefficient increases with the increasing values of $\lambda$, and the mass transfer coefficient decreases with the increasing values of $E$ and $w$. It is observed from this table that this is an increasing effect of suction parameter $v_{0}$ on the skin friction coefficient and heat and mass transfer coefficients. Finally, the effects of heat generation/absorption coefficient $(\gamma)$ on the dimensionless Nusselt number $N_{u}$ are shown in Table 1. The Nusselt number increases with the increasing values of the heat absorption coefficient $(\gamma<0)$. Because the heat absorption increases, the disk tends to supply more energy through its wall to maintain the constant temperature of the wall, and hence the Nusselt number increases. Opposite effects are found for the increasing values of the heat generation coefficient $(\gamma>0)$.
5.7. Comparison between Our Numerical Result and the Analytical Result of Bestman [8]. Bestman studied steady natural convective boundary layer flow with large suction. He solved his problem analytically by employing the perturbation technique proposed by Singh and Dikshit [9]. In our present work, we took $A=0$ in (14) for considering steady flow. The values of the suction parameter $v_{0}^{2}=10$ are taken to see the effects of large suction. $G_{r}=G_{m}=1.0, \lambda=5, M=E_{c}=\gamma=$ $0, E=5$, and $w=1$ are also chosen with a view to compare our numerical results with the analytical results of Bestman [8]. The comparison of velocity profiles as seen in Figure 12 highlights the validity of the numerical computations adapted in the present investigation.

\section{Conclusions}

In this paper, we investigate the effects of chemical reaction rate and Arrhenius activation energy on an unsteady natural convection heat and mass transfer boundary layer flow past a flat porous plate in the presence of uniform magnetic field viscous dissipation and heat generation/absorption.

The Nachtsheim-Swigert [16] iteration technique based on the sixth-order Range-Kutta and shooting methods has been employed to complete the integration of the resulting solutions.

The following conclusions can be drawn as a result of the computations.

(1) Velocity increases with increasing values of $G_{r}$ for the cooling of the plate, and the negative increase in the Grashof number leads to the decrease in the velocity field. That is, for heating the plate, the effects of the Grashof number $G_{r}$ on the velocity field have also opposite effects, as compared to the cooling of the plate.

(2) Solutal Grashof number $G_{m}>0$ that corresponds to the chemical species concentration in the free stream region is less than the concentration at the boundary surface. It is observed that the velocity profile increases with the increasing values of Solutal Grashof number $G_{m}$.

(3) We observe that the velocity field decreases due to magnetic interaction parameter $M$. This is because the application of a transverse magnetic field normal to the flow direction will result in a resistive force (Lorentz force) similar to a drag force which tends to resist the fluid flow and thus reduces its velocity.

(4) Eckert number $\left(E_{c}\right)$ has marked effect on velocity and temperature fields. The velocity and the temperature profiles increase with the increasing values of $E_{c}$.

(5) Increase in $\lambda$ leads to the decrease in the concentration profiles. It is observed from (12) that increasing temperature frequently causes a marked increase in the rate of reactions $\lambda$. The velocity profile slightly increases with the increasing values of $\lambda$.

(6) Increase in activation energy (E) leads to increasing the concentration, temperature, and velocity profiles. 
For small changes of activation energy that must occur to convert the reactants into the product of the reaction, energy must be invested to begin breaking the reactant bond so that product bond be can formed.

(7) For strong suction $\left(v_{0}>0\right)$, the velocity, the temperature, and the concentration profiles decay rapidly away from the surface. As for the injection $\left(v_{0}>0\right)$, it is observed that the boundary layer is increasingly blown away from the plate to form an interlayer between the injection and the outer flow regions.

(8) The Nusselt number increases with the increasing values of the heat absorption coefficient $(\gamma<0)$. Because the heat absorption increases, the disk tends to supply more energy through its wall to maintain the constant temperature of the wall, and hence the Nusselt number increases.

\section{Nomenclature}

$(u, v)$ : components of velocity field

$c_{p}$ : $\quad$ specific heat at constant pressure

$N_{u}$ : Nusselt number

$P_{r}$ : Prandtl number

$T: \quad$ temperature of the flow field

$T_{\infty}$ : temperature of the fluid at infinity

$T_{w}$ : temperature at the plate

$f: \quad$ dimensionless similarity functions

$E_{a}: \quad$ the activation energy

$k$ : $\quad$ the Boltzmann constant

$S_{c}$ : Schmidt number

$E: \quad$ the nondimensional activation energy

$G_{r}: \quad$ Grashof number

$G_{m}: \quad$ modified (Solutal) Grashof number.

\section{Greek Symbols}

$\begin{array}{ll}\theta: & \text { Dimensionless temperature } \\ \phi: & \text { dimensionless concentration } \\ \eta: & \text { dimensionless similarity variable } \\ \delta: & \text { scale factor } \\ \nu: & \text { kinematic viscosity } \\ \rho: & \text { density of the fluid } \\ \kappa: & \text { thermal conductivity } \\ \tau: & \text { shear stress } \\ \mu: & \text { fluid viscosity } \\ \lambda^{2}: & \text { the nondimensional chemical reaction } \\ & \text { rate constant } \\ \beta \text { and } \beta^{*}: & \text { the coefficients of volume expansions for } \\ & \text { temperature, and concentration }\end{array}$

\section{References}

[1] M. Tencer, J. S. Moss, and T. Zapach, "Arrhenius average temperature: the effective temperature for non-fatigue wearout and long term reliability in variable thermal conditions and climates," IEEE Transactions on Components and Packaging Technologies, vol. 27, no. 3, pp. 602-607, 2004.

[2] C. Truesdell, "Sulle basi della thermomeccanica," Accademia Nazionale del Lincei, Rendiconti della Classe di Scienze Fisiche, vol. 22, no. 8, pp. 33-38, 1957.

[3] C. Truesdell, "Sulle basi della thermomeccanica," Accademia Nazionale del Lincei, Rendiconti della Classe di Scienze Fisiche, vol. 22, no. 8, pp. 158-166, 1957.

[4] N. Mills, "Incompressible mixtures of newtonian fluids," International Journal of Engineering Science, vol. 4, no. 2, pp. 97-112, 1966.

[5] C. E. Beevers and R. E. Craine, "On the determination of response functions for a binary mixture of incompressible newtonian fluids," International Journal of Engineering Science, vol. 20, no. 6, pp. 737-745, 1982.

[6] A. Al-Sharif, K. Chamniprasart, K. R. Rajagopal, and A. Z. Szeri, "Lubrication with binary mixtures: liquid-liquid emulsion," Journal of Tribology, vol. 115, no. 1, pp. 46-55, 1993.

[7] S. H. Wang, A. Al-Sharif, K. R. Rajagopal, and A. Z. Szeri, "Lubrication with binary mixtures: liquid-liquid emulsion in an EHL conjunction," Journal of Tribology, vol. 115, no. 3, pp. 515$522,1993$.

[8] A. R. Bestman, "Natural convection boundary layer with suction and mass transfer in a porous medium," International Journal of Energy Research, vol. 14, no. 4, pp. 389-396, 1990.

[9] A. K. Singh and C. K. Dikshit, "Hydromagnetic flow past a continuously moving semi-infinite plate for large suction," Astrophysics and Space Science, vol. 148, no. 2, pp. 249-256, 1988.

[10] A. R. Bestman, "Radiative heat transfer to flow of a combustible mixture in a vertical pipe," International Journal of Energy Research, vol. 15, no. 3, pp. 179-184, 1991.

[11] M. A. Alabraba, A. R. Bestman, and A. Ogulu, "Laminar convection in binary mixture of hydromagnetic flow with radiative heat transfer, I," Astrophysics and Space Science, vol. 195, no. 2, pp. 431-439, 1992.

[12] R. Kandasamy, K. Periasamy, and K. K. S. Prabhu, "Effects of chemical reaction, heat and mass transfer along a wedge with heat source and concentration in the presence of suction or injection," International Journal of Heat and Mass Transfer, vol. 48, no. 7, pp. 1388-1394, 2005.

[13] O. D. Makinde, P. O. Olanrewaju, and W. M. Charles, "Unsteady convection with chemical reaction and radiative heat transfer past a flat porous plate moving through a binary mixture," Afrika Matematika, vol. 21, no. 1, pp. 1-17, 2011.

[14] Kh. A. Maleque, "Unsteady natural convection boundary layer heat and mass transfer flow with exothermic chemical reactions," Journal of Pure and Applied Mathematics, vol. 9, no. 1, pp. 17-41, 2013.

[15] Kh. A. Maleque, "Effects of combined temperature- and depthdependent viscosity and hall current on an unsteady MHD laminar convective flow due to a rotating disk," Chemical Engineering Communications, vol. 197, no. 4, pp. 506-521, 2010.

[16] P. R. Nachtsheim and P. Swigert, "Satisfaction of asymptotic boundary conditions in numerical solution of system of nonlinear of boundary layer type," NASA TN-D3004, 1965. 

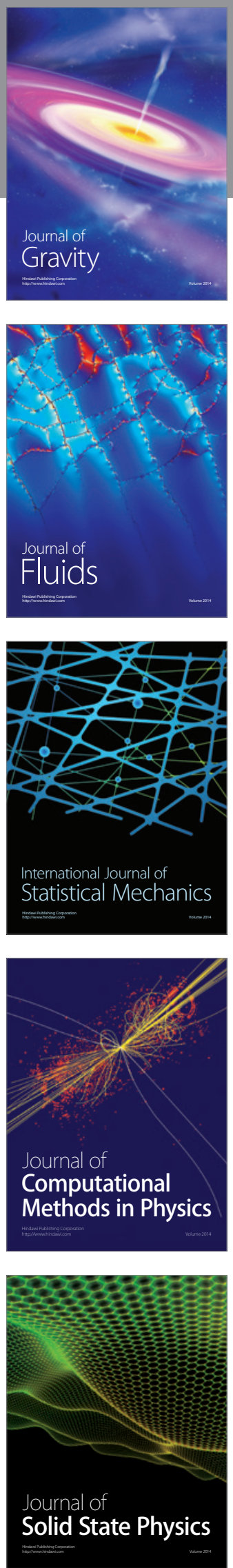

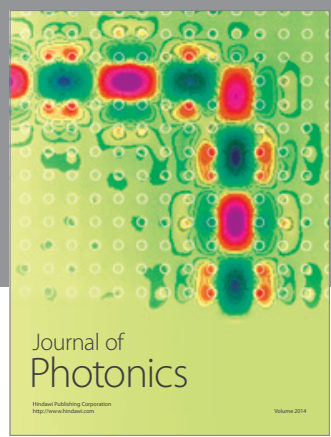

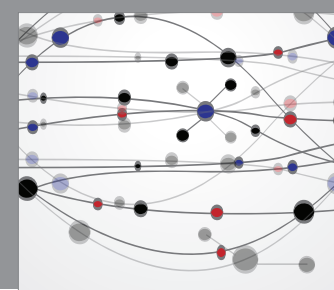

The Scientific World Journal

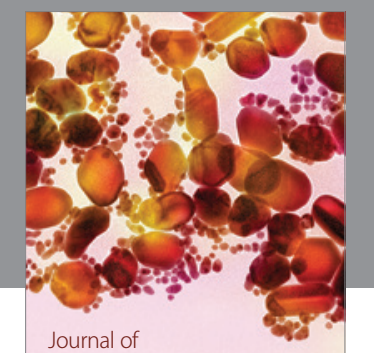

Soft Matter
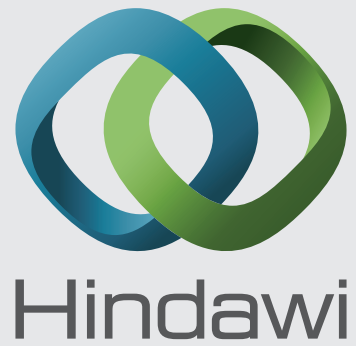

Submit your manuscripts at

http://www.hindawi.com
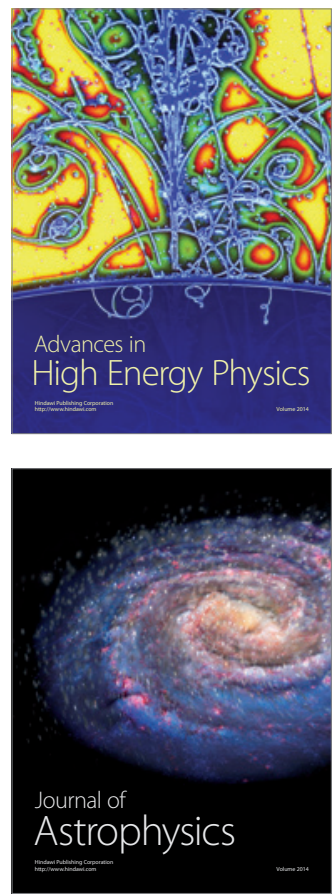
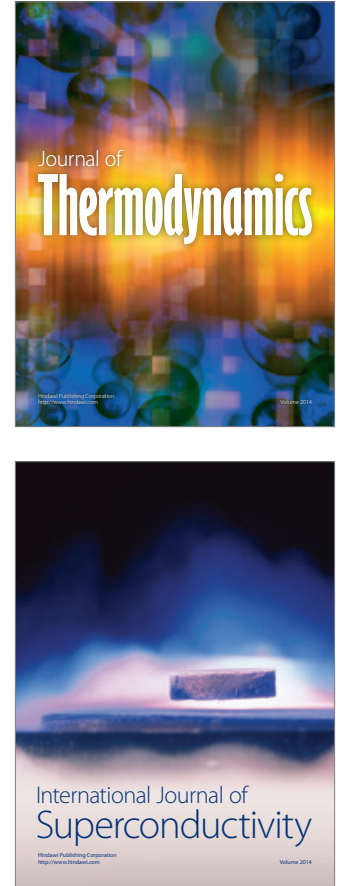
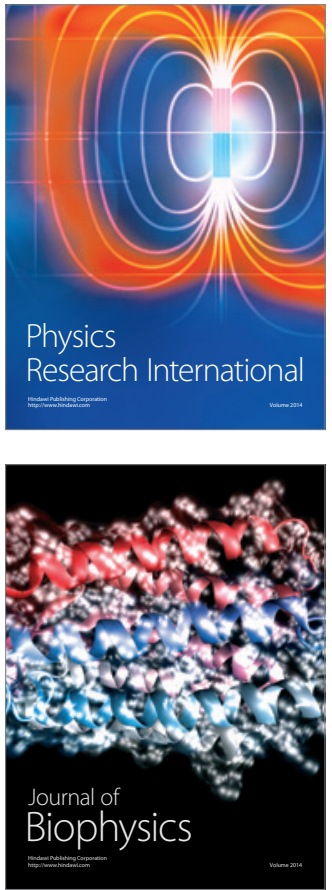
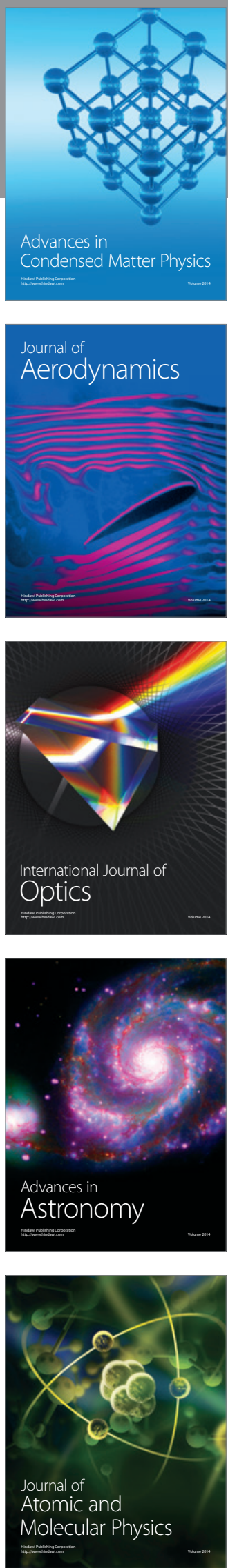